\title{
Impact of Angiotensin Receptor Blockers, Beta Blockers, Calcium Channel Blockers and Thiazide Diuretics on Survival of Ovarian Cancer Patients
}

\author{
Min Ae Cho, MD 1 \\ Soo Young Jeong, MD \\ Insuk Sohn, MD, PhD2 \\ Myeong-Seon Kim, MD \\ Jun Hyeok Kang, MD \\ E Sun Paik, MD, PhD' \\ Yoo-Young Lee, MD, $\mathrm{PhD}{ }^{1}$ \\ Chel Hun Choi, MD, PhD'
}

\begin{abstract}
Purpose
We investigated the impact of four types of antihypertensive medications, angiotensin receptor blockers (ARBs), beta blockers (BBs; both selective and non-selective), calcium channel blockers (CCBs), and thiazide diuretics (TDs) on survival outcomes in epithelial ovarian cancer (EOC).
\end{abstract}

\section{Materials and Methods}

A single-institutional retrospective chart review of 878 patients with EOC was performed. Survival was compared according to use of the four antihypertensive medications during primary treatment. Propensity score matching (ratio 1:3) was performed to control possible associated covariates, such as age, International Federation of Gynecology and Obstetrics stage, residual status after primary debulking surgery, and co-morbidity.

\section{Results}

Among 878 patients, 56 patients (6.4\%) were ARB users, 62 (7.1\%) were BB users, 107 (12.2\%) were CCBs users and 32 (3.6\%) used TDs. Median progression-free survival (PFS) for ARB, BB, and CCB users was 37.8, 27.2, and 23.6 months compared with 33.6 months for non-users. ARB was associated with $35 \%$ decreased risk of disease progression (hazard ratio [HR], 0.65 ; $95 \%$ confidence interval [Cl], 0.42 to $0.99 ; p=0.046$ ) in multivariate analysis. After propensity score matching, median PFS for ARB users was 37.8 months and ARB use remained to be associated with lower recurrence rate in univariate $(\mathrm{p}=0.035)$ and $\mathrm{mul}-$ tivariate analysis ( $\mathrm{HR}, 0.60 ; 95 \% \mathrm{Cl}, 0.39$ to $0.93 ; \mathrm{p}=0.022$ ).

\section{Conclusion}

In this study, ARBs use during primary treatment is associated with lower recurrence in EOC patients. However, CCBs, BBs, and TDs did not show beneficial impact.

Tel: 82-2-3410-3545

Fax: 82-2-3410-0630

E-mail: chelhun.choi@samsung.com

Received October 21, 2019

Accepted January 6, 2020

Published Online January 16, 2020

Key words

Ovarian neoplasms, Antihypertensive agents,

Angiotensin II type 1 receptor blockers,

${ }^{*}$ Min Ae Cho and Soo Young Jeong contributed Adrenergic beta-antagonists, Calcium channel blockers, Thiazides

\section{Introduction}

Despite the many advances in its treatment and management, epithelial ovarian cancer (EOC) is still one of the leading causes of death in gynecologic malignancies. Being undoubtedly the most critical among gynecologic cancers, EOC shows a tendency of higher incidence in developed countries. The age-standardized incidence of ovarian cancer in Korea has been rising, reaching as much as 6.3 per 100,000 in 2015 [1]. Even with standardized management of debulking surgery followed by systemic chemotherapy has improved survival rates, 5-year survival rate was reported to be $64 \%$ in 2016, with the poorest outcome among gynecologic malignancies [2].

Therefore, more clinical treatment options are necessary to minimize the disease burden of ovarian cancer. While modest advances in chemotherapy have been made in the past decades, systemic options for EOC patients remain somewhat limited. As such, there is great interest in identifying 
potential combination therapies that may target other aspects of ovarian tumor biology to increase the effectiveness of systemic therapy.

In this circumstance, several antihypertensive medications, which are one of the most widely used drugs, are thought to improve EOC survival rate. Angiotensin II, a biologically active peptide of the renin-angiotensin system, has been shown to be involved in cell proliferation, migration and angiogenesis in the molecular etiologies of other neoplasms [3]. As such, angiotensin II receptor blockers (ARBs) may have potential therapeutic benefits by blocking the downstream effects of angiotensin II not only on cancer cells, but also on endothelial cells within the tumor and stroma $[4,5]$. Indeed, several in vitro studies have reported that losartan (an ARB) can markedly suppress pancreatic cancer cell proliferation by triggering apoptosis and reducing vascular endothelial growth factor (VEGF) expression, a critical component of angiogenesis [6,7]. Moreover, studies focusing on drug delivery have shown that losartan enhances drug delivery and potentiates chemotherapy by inhibiting stromal collagen and hyaluronic synthesis, thereby decompressing tumor blood vessels and reducing solid tumor stress $[8,9]$. In fact, ovarian cancer cells have higher concentrations of angiotensin II type 1 and 2 receptor compared to borderline or benign ovarian tumor [10]. Corresponding with pathophysiologic models, angiotensin II type 1 receptor expression was correlated with tumor angiogenesis and poor prognosis in ovarian cancer [11]. Preclinical trials suggest that ARBs, which are commonly used as antihypertensive and renal protective medications, have favorable impact on cancer prognosis $[12,13]$.

Several retrospective cohort studies showed potency of beta-blockers (BBs) in reducing ovarian cancer recurrence and prolonging PFS [14,15]. In some of those studies, only non-selective beta blockers (NSBB) were proved to be effective for prolonging EOC survival $[14,16]$. Meanwhile, data on the impact of other antihypertensive agents, including calcium channel blockers (CCBs) and thiazide diuretics (TDs), on EOC survival rate is sparse [17].

We can speculate that primary EOC patients treated with antihypertensive drugs would have better survival rates. However, only a few clinical investigations to date have investigated the potential effect of ARBs for survival outcomes of ovarian cancer patients [9]. Also, there are not many studies for comparison between different types of blood pressure medications $[9,17]$. Given the potential role of ARBs in the treatment of patients with EOC, clinical data on the impact of ARB use in ovarian cancer is important. Therefore, the objective of the current study was to evaluate the association of ARB use with survival among EOC patients along with BBs, CCBs, and TDs.

\section{Materials and Methods}

\section{Patient selection}

We reviewed all patients with primary EOC who were treated at Samsung Medical Center from January 2001 to December 2014. We retrospectively obtained data from Samsung Medical Center electronic medical records and enrolled patients depending on the inclusion criteria as follows: (1) primary EOC with International Federation of Gynecology and Obstetrics (FIGO) stage I to IV disease; (2) patients who were treated with primary debulking surgery (PDS) with adjuvant chemotherapy for primary treatment. The exclusion criteria were as follows: (1) patients who had neo-adjuvant chemotherapy with interval debulking surgery; (2) histology with non-EOC. In this study, a total of $878 \mathrm{EOC}$ patients were investigated.

The data of patients' age at diagnosis, tumor stage, histologic type, residual status, use of antihypertensive drug, and comorbidities were obtained. 'Comorbidity' was defined as having any of hypertension, diabetes, cerebrovascular accident, dyslipidemia, or cardiovascular disease.

\section{Treatment and follow-up}

For initial work-up before primary treatment, patients were routinely examined by imaging studies. The status of residual disease after PDS was determined by the largest diameter of residual disease and categorized as follows: $<1$ $\mathrm{cm}$ residual disease for optimal and $\geq 1 \mathrm{~cm}$ residual disease for suboptimal operation. After PDS, all enrolled patients had been treated with adjuvant chemotherapy. The combination chemotherapy, consisted of paclitaxel $\left(175 \mathrm{mg} / \mathrm{m}^{2}\right)$ and carboplatin (area under the curve $\times 5$ ), was initiated routinely within 2 weeks of surgery. The standard regimen of continued every 3 weeks for six cycles. The institutional protocol recommends early start of adjuvant chemotherapy unless complications develop, but there may have been variations of the intervals and the number of cycles depending on the patient's situation. After primary treatment, all patients received follow-up procedures. Physical examination and tumor markers were performed every 3 months for the first 2 years and every 6 months for the next 3 years. Imaging studies were performed every 6-12 months for the first 2 years and every 12 months for the next 3 years. For survival analysis, progression-free survival (PFS) was evaluated from the date of primary surgery to the period of recurrence/ progression or the time of the last follow-up visit. The cause of death of all patients were not known accurately and treatment regimen was different for each person at the time of relapse, so, overall survival was not evaluated in this study. 
Table 1. Patient characteristics of study cohorts

\begin{tabular}{|c|c|c|c|c|c|c|}
\hline \multirow{2}{*}{ Characteristic } & \multicolumn{2}{|c|}{$\begin{array}{l}\text { Angiotensin receptor blockers } \\
\text { (ARBs) }\end{array}$} & \multicolumn{2}{|c|}{$\begin{array}{c}\text { Beta blockers } \\
\text { (BBs) }\end{array}$} & \multicolumn{2}{|c|}{$\begin{array}{l}\text { Calcium channel blockers } \\
\text { (CCBs) }\end{array}$} \\
\hline & $\begin{array}{l}\text { Without ARB } \\
(n=822)\end{array}$ & $\begin{array}{c}\text { With ARB } \\
(\mathrm{n}=56)\end{array}$ & $\begin{array}{l}\text { Without BB } \\
\quad(n=816)\end{array}$ & $\begin{array}{c}\text { With BB } \\
(n=62)\end{array}$ & $\begin{array}{l}\text { Without CCB } \\
\quad(n=771)\end{array}$ & $\begin{array}{c}\text { With CCB } \\
(\mathrm{n}=\mathbf{1 0 7})\end{array}$ \\
\hline Age (yr) & $50.5(44.0-60.0)$ & $61.0(53.0-67.5)$ & $51.0(44.0-60.0)$ & $55.0(47.0-60.0)$ & $50.0(43.0-59.0)$ & $60.0(52.0-65.0)$ \\
\hline \multicolumn{7}{|l|}{ Type } \\
\hline Serous & $513(62.4)$ & $40(71.4)$ & $514(63.0)$ & $39(62.9)$ & $469(60.8)$ & $84(78.5)$ \\
\hline Non-serous & $309(37.6)$ & $16(28.6)$ & $302(37.0)$ & $23(37.1)$ & $302(39.2)$ & $23(21.5)$ \\
\hline \multicolumn{7}{|l|}{ FIGO stage } \\
\hline I & $189(23.0)$ & 11 (19.6) & $187(22.9)$ & $13(21.0)$ & $188(24.4)$ & $12(11.3)$ \\
\hline II & $86(10.5)$ & $5(8.9)$ & $85(10.4)$ & $6(9.7)$ & $78(10.1)$ & $13(12.1)$ \\
\hline III & $461(56.0)$ & $32(57.2)$ & $461(56.5)$ & $32(51.6)$ & $428(55.5)$ & $65(60.7)$ \\
\hline IV & $86(10.5)$ & $8(14.3)$ & $83(10.2)$ & $11(17.7)$ & $77(10.0)$ & $17(15.9)$ \\
\hline \multicolumn{7}{|c|}{ Platinum resistance } \\
\hline Sensitive & $698(84.9)$ & $52(92.9)$ & $695(85.2)$ & $55(90.6)$ & $661(85.7)$ & $89(83.2)$ \\
\hline Resistant & $124(15.1)$ & $4(7.1)$ & $121(14.8)$ & $7(9.4)$ & $110(14.3)$ & $18(16.8)$ \\
\hline \multicolumn{7}{|l|}{ Residual status } \\
\hline No residual & $309(37.6)$ & $16(28.6)$ & $299(36.6)$ & $26(41.9)$ & $297(38.5)$ & $28(26.2)$ \\
\hline$<1 \mathrm{~cm}$ & $316(38.4)$ & $18(32.1)$ & $311(38.1)$ & $23(37.1)$ & $287(37.2)$ & $47(43.9)$ \\
\hline$\geq 1 \mathrm{~cm}$ & $197(24.0)$ & $22(39.3)$ & $206(25.3)$ & $13(21.0)$ & $187(24.3)$ & $32(29.9)$ \\
\hline \multicolumn{7}{|c|}{$B R C A 1 / 2$ mutation } \\
\hline No & $47(63.5)$ & $3(100)$ & $47(65.3)$ & $3(60.0)$ & $44(64.7)$ & $6(66.7)$ \\
\hline Yes & $27(36.5)$ & 0 & $25(34.7)$ & $2(40.0)$ & $24(35.3)$ & $3(33.3)$ \\
\hline \multicolumn{7}{|l|}{ Comorbidity } \\
\hline No & $702(85.4)$ & $4(7.1)$ & $662(81.1)$ & $44(71.0)$ & $685(88.8)$ & $21(19.6)$ \\
\hline Yes & $120(14.6)$ & $52(92.9)$ & $154(18.9)$ & $18(29.0)$ & $86(11.2)$ & $86(80.4)$ \\
\hline \multicolumn{7}{|l|}{ Hypertension } \\
\hline No & $718(87.6)$ & $5(8.9)$ & $677(83.2)$ & $46(74.2)$ & $699(90.9)$ & $24(22.4)$ \\
\hline Yes & $102(12.4)$ & $51(91.1)$ & $137(16.8)$ & $16(25.8)$ & $70(9.1)$ & $83(77.6)$ \\
\hline \multicolumn{7}{|l|}{ Diabetes } \\
\hline No & $573(94.4)$ & $32(74.4)$ & $567(93.7)$ & $38(84.4)$ & $528(93.6)$ & $77(89.5)$ \\
\hline Yes & $34(5.6)$ & $11(25.6)$ & $38(6.3)$ & $7(15.6)$ & $36(6.4)$ & $9(10.5)$ \\
\hline \multicolumn{7}{|c|}{ Cerebrovascular accident } \\
\hline No & $600(98.7)$ & $35(81.4)$ & $592(97.7)$ & $43(95.6)$ & $553(97.9)$ & $82(95.3)$ \\
\hline Yes & $8(1.3)$ & $8(18.6)$ & $14(2.3)$ & $2(4.4)$ & $14(2.1)$ & $4(4.7)$ \\
\hline \multicolumn{7}{|c|}{ Cardiovascular disease } \\
\hline No & $593(97.5)$ & $36(83.7)$ & $586(96.7)$ & 43 (95.6) & $551(97.5)$ & $78(90.7)$ \\
\hline Yes & $15(2.5)$ & 7 (16.3) & $20(3.3)$ & $2(4.4)$ & $14(2.5)$ & $8(9.3)$ \\
\hline \multicolumn{7}{|l|}{ Dyslipidemia } \\
\hline No & $586(96.4)$ & 35 (81.4) & $582(96.0)$ & $39(86.7)$ & $539(95.4)$ & $82(95.3)$ \\
\hline Yes & $22(3.6)$ & $8(18.6)$ & $24(4.0)$ & $6(13.3)$ & $26(4.6)$ & $4(4.7)$ \\
\hline
\end{tabular}

Values are presented as median (range) or number $(\%)$.

\section{Drug use}

The use of drug was defined as documented prescription of $\mathrm{ARB} / \mathrm{BB} / \mathrm{CCB} / \mathrm{TD}$ for 6 months following diagnosis or operation of EOC. Both the patients who were taking medicine prior to the diagnosis of EOC and new prescriptions were included. We defined non-users as those with no prescription records of $\mathrm{ARB} / \mathrm{BB} / \mathrm{CCB} / \mathrm{TD}$ before and within 6 months of diagnosis or operation of EOC. Every patient who may have been taking the medicine previously, but not during the 6 months following diagnosis of EOC, were categorized as non-user. 


\section{Statistical analysis}

We used the summary statistics to describe the data. Median range or standard deviations were used for continuous variables. Mann-Whitney test for comparing median values and Student's $t$ test for comparing mean values were used after the Shapiro-Wilks test to confirm normal distributions. We presented categorical variables as percentages. We used Fisher exact test or chi-square test for analyzing the distribution of characteristics according to use of antihypertensives. Analyses for survival curves were performed by the Kaplan-Meier method and comparison was done by the logrank test. We used the Cox proportional hazards model to perform univariate and multivariate analyses for evaluation of the prognostic significance of the use of antihypertensives and other clinicopathological features. All p-values were two-sided, and we considered p-values of less than 0.05 as statistically significant. We performed statistical analyses using R 3.0.3 (Vienna, Austria; http:// www.R-project.org).

After the total cohort analyses, propensity score matching was performed to further investigate the characteristics of the patients. Drug users were 1:3 matched with the closest propensity patients without drugs according to age, FIGO stage, residual disease status after PDS, BRCA mutation status, and comorbidity (performed with $\mathrm{R}$ using the MatchIt package). The propensity scores were calculated using a multivariable logistic regression model based on factors that demonstrated significant differences between the two groups in the total cohort.

\section{Ethical statement}

The study was approved by the Institutional Review Board of the Samsung Medical Center (IRB No. 2019-05-129). Given the retrospective nature of the study, direct informed consent from the women was not necessary as per the ethical guidelines.

\section{Results}

\section{Patient characteristics}

The clinical features of the included patients are shown in Table 1 and S1 Table. Among 878 ovarian cancer patients, 56 patients $(6.4 \%)$ were ARB users, 62 patients $(7.1 \%)$ were BB users, 107 patients $(12.2 \%)$ were CCB users, 32 patients $(3.6 \%)$ were TD users and 687 patients $(78.2 \%)$ were non-users. Median age at diagnosis was 51.9 years for those patients without any antihypertensive drugs. Median age of diagno-
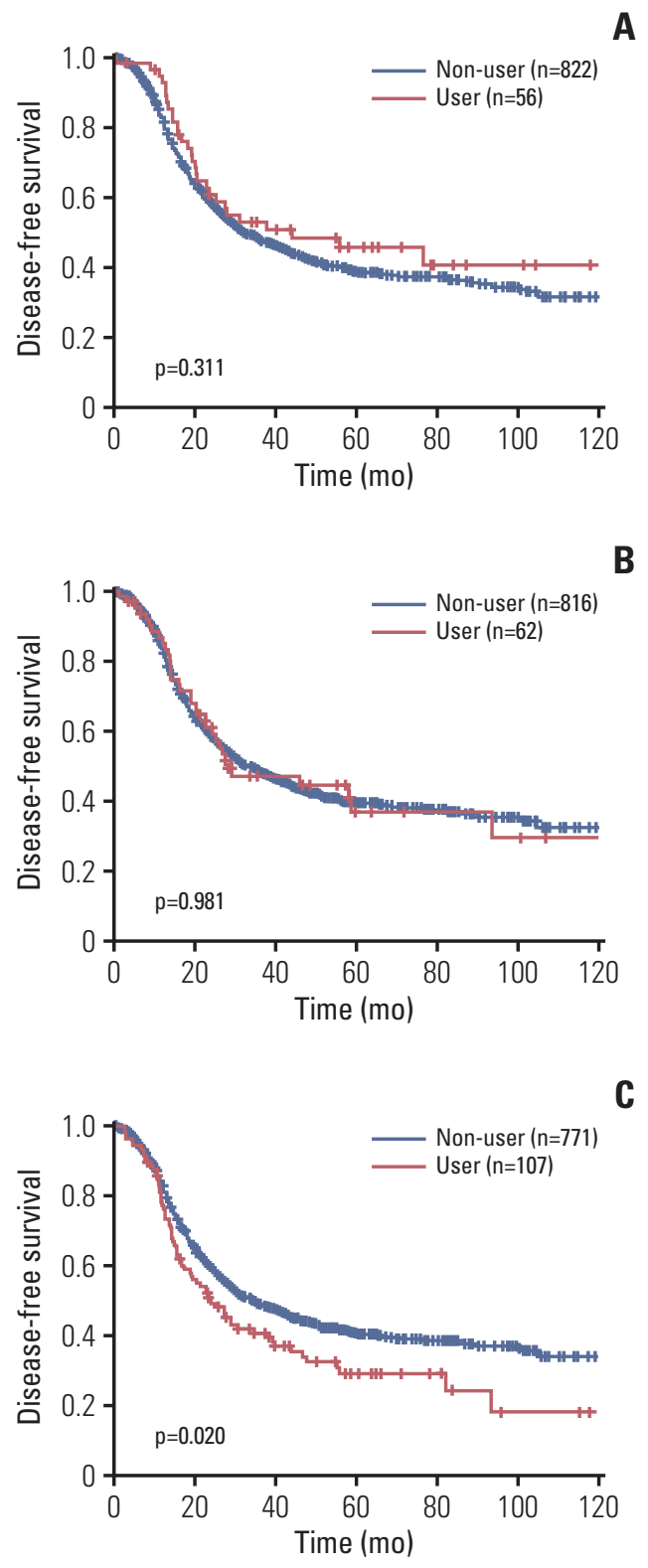

Fig. 1. Kaplan-Meier curve for progression-free survival according to angiotensin receptor blocker (A), beta blocker (B), and calcium channel blocker (C).

sis was $61,55,60$, and 66 years respectively for ARB, BB, $\mathrm{CCB}$, and TD users. There were no significant difference in histologic type and FIGO stage between users and non-users of ARBs and BBs, however, most CCB users had serous type (60.8\% of non-users vs. $78.5 \%$ of users) and advanced stage (65.5\% of non-users vs. $76.6 \%$ of users). Platinum resistance between each group of four drugs did not show statistical difference. Cytoreduction status after PDS revealed more frequent suboptimal operation for ARB users $(24.0 \%$ of non- 
Table 2. Multivariate Cox proportional hazards analysis for progression-free survival $(\mathrm{n}=878)$

\begin{tabular}{lcr} 
Variable & HR $(95 \%$ CI $)$ & p-value \\
\hline Age $(>50$ yr) & $1.06(0.87-1.30)$ & 0.534 \\
FIGO stage (III / IV) & $5.32(3.96-7.13)$ & $<0.001$ \\
Residual status $(>1 \mathrm{~cm})$ & $1.45(1.18-1.76)$ & $<0.001$ \\
Comorbidity (yes) & $1.05(0.81-1.35)$ & 0.728 \\
ARBs (use) & $0.65(0.42-0.99)$ & 0.046 \\
BBs (use) & $0.95(0.67-1.35)$ & 0.770 \\
CCBs (use) & $1.20(0.87-1.65)$ & 0.257 \\
\hline
\end{tabular}

HR, hazard ratio; CI, confidence interval; FIGO, International Federation of Gynecology and Obstetrics; ARBs, angiotensin-receptor blockers; BBs, beta blockers; CCBs, calcium channel blockers.

users vs. $39.3 \%$ of users, $\mathrm{p}=0.036), \mathrm{CCB}$ users $(24.3 \%$ of nonusers vs. $29.9 \%$ of users, $\mathrm{p}=0.045)$ and TD users $(24.5 \%$ of non-users vs. $37.5 \%$ of users, $\mathrm{p}=0.183)$. Not many patients were performed the BRCA test with blood or specimen and just 77 patients in this study had been done this test. There is no significant difference of $B R C A$ mutation status between drug users and non-users. Comorbidities were highly reported in patients those who take antihypertensive agents; $14.6 \%$ vs. $92.9 \%$ for ARBs ( $<<0.001$ ), 18.9 vs. $29.0 \%$ for BBs ( $p<$ $0.001), 11.2$ vs. $80.4 \%$ for CCBs ( $<<0.001)$, and $16.9 \%$ vs. 90.6\% for TDs $(\mathrm{p}<0.001)$.

\section{Survival}

In total, median PFS was 33.9 months for ovarian cancer patients in this study. At the time of this analysis, 470 of 878 enrolled patients $(53.5 \%)$ had experienced relapse and 277 $(31.5 \%)$ had died after a median observation time. Among those patients, 37 had died from causes other than EOC. The median survival of ARB users was the longest with PFS of 37.8 months. The median PFS of TD users was 32.1 months, which was the shortest among patient groups.

Kaplan-Meier curves for PFS in each antihypertensive drug are shown in Fig. 1. The use of ARBs seemed to be associated with lower recurrence rate but was not statistically significant $(\mathrm{p}=0.311)$. BBs did not show statistically significant impact on PFS of ovarian cancer patients $(\mathrm{p}=0.981)$. Subgroup analysis for BB users to verify difference between selective and NSBB was done. Both selective beta blockers $(\mathrm{p}=0.647)$ and NSBB $(\mathrm{p}=0.645)$ did not show significant relevance with ovarian cancer progression (S2B and S2C Fig.). The patients who were taking CCBs had higher frequency of recurrence significantly $(\mathrm{p}=0.020)$. In S2A Fig., no association was found between TD usage and disease recurrence $(p=$
0.406).

Multivariate Cox regression analysis was calculated in order to assess independent prognostic factors for recurrence outcome (Table 2). Age, stage, and cytoreduction status, which are widely accepted as critical prognostic factors of EOC, were included in the analysis to adjust for survival outcomes. The presence of comorbidities was also evaluated to assess the effect on survival in the overall group.

Advanced stage and suboptimal PDS were associated with higher recurrence in this study. ARBs had favorable impact on recurrence (hazard ratio [HR], $0.65 ; 95 \%$ confidence interval [CI], 0.42 to $0.99 ; p=0.046)$. However, usage of BBs, CCBs was not a prognostic factor in recurrence. The HR for recurrence were 0.95 ( $95 \% \mathrm{CI}, 0.67$ to $1.35 ; \mathrm{p}=0.770)$ for $\mathrm{BB}$ users and 1.20 (95\% CI, 0.88 to $1.65 ; \mathrm{p}=0.257)$ for CCB users.

\section{Survival after matching}

To reduce the selection bias when comparing two groups of patients, additional propensity score matching was performed with R using the MatchIt package (S3 Fig. for distribution of propensity scores). With nearest-neighbor 1:3 ratio according to age, FIGO stage, histologic type, residual disease status after PDS, platinum resistance, and comorbidity, each patient group with or without each type of antihypertensive medication were matched. The analysis for ARB included a total of 224 patients, 56 users and 168 non-users. Matching for BBs and CCBs included 248 (with BB, 62; without $\mathrm{BB}, 186$ ) and 428 (with $C C B, 107$; without CCB, 321), respectively.

Regarding clinicopathological characteristics, each matching was successful without significant differences between paired groups for stage, histologic type, residual disease status, and platinum resistance except for comorbidity status. (Table 3 for ARB, BB, and CCB; S4 Table for TD, non-selective beta blockers [SBB], and NSBB) Because of difference in comorbidity status in ARB and CCB groups, we analyzed multivariate Cox proportional hazard analysis and KaplanMeier curves of only co-morbidity patients.

In Fig. 2, patients with ARB still showed better outcomes for PFS (median PFS of users vs. non-users, 37.8 months vs. 27.4 months; $\mathrm{p}=0.035$ ). However, neither $\mathrm{BB}, \mathrm{CCB}$ nor $\mathrm{TD}$ was found to be associated with $\mathrm{EOC}$ recurrence. The median PFS was 27.2 months for BB users and 28.0 months for nonusers, and there was no significant association between usage of BBs and progression ( $\mathrm{p}=0.678$ ). The patients with CCBs had no relationship in recurrence with this matched analysis; median PFS was 27.5 months for CCB users compared with 27.9 months for non-users $(\mathrm{p}=0.797)$. In multivariate Cox regression analysis with this matched group (Table 4), ARBs remained a significant prognostic factor with $40 \%$ risk reduction for recurrence (HR, $0.60 ; 95 \% \mathrm{CI}, 0.39$ to $0.93 ; \mathrm{p}=0.022)$. 


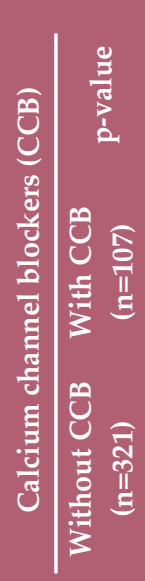

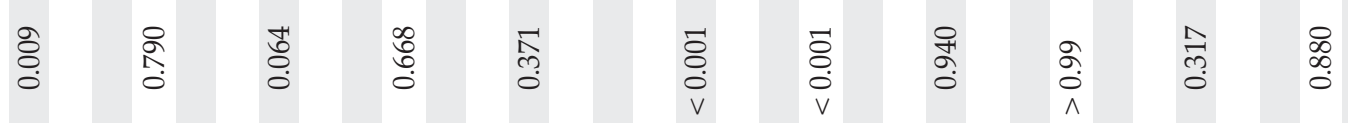

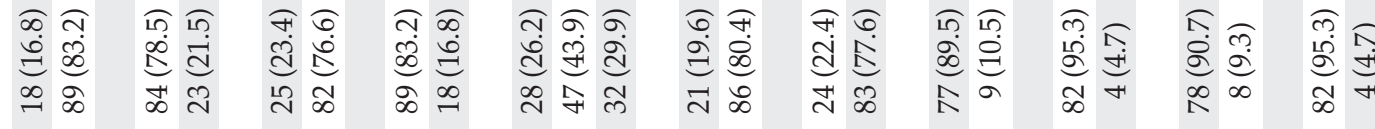

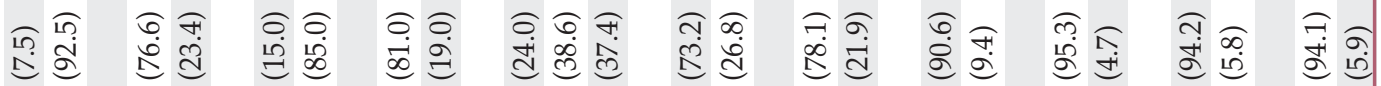

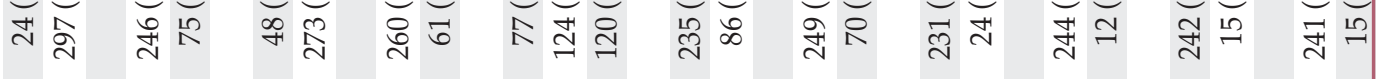

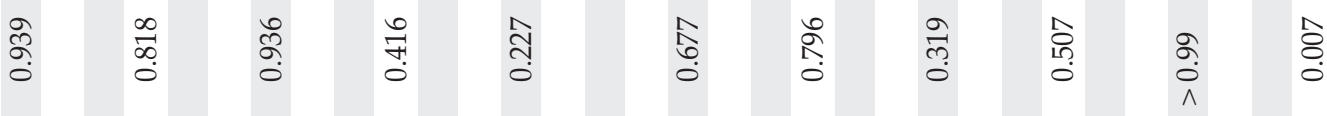

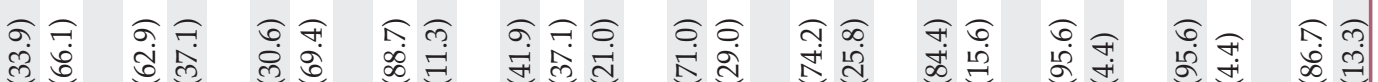
ป⿻ ने के 

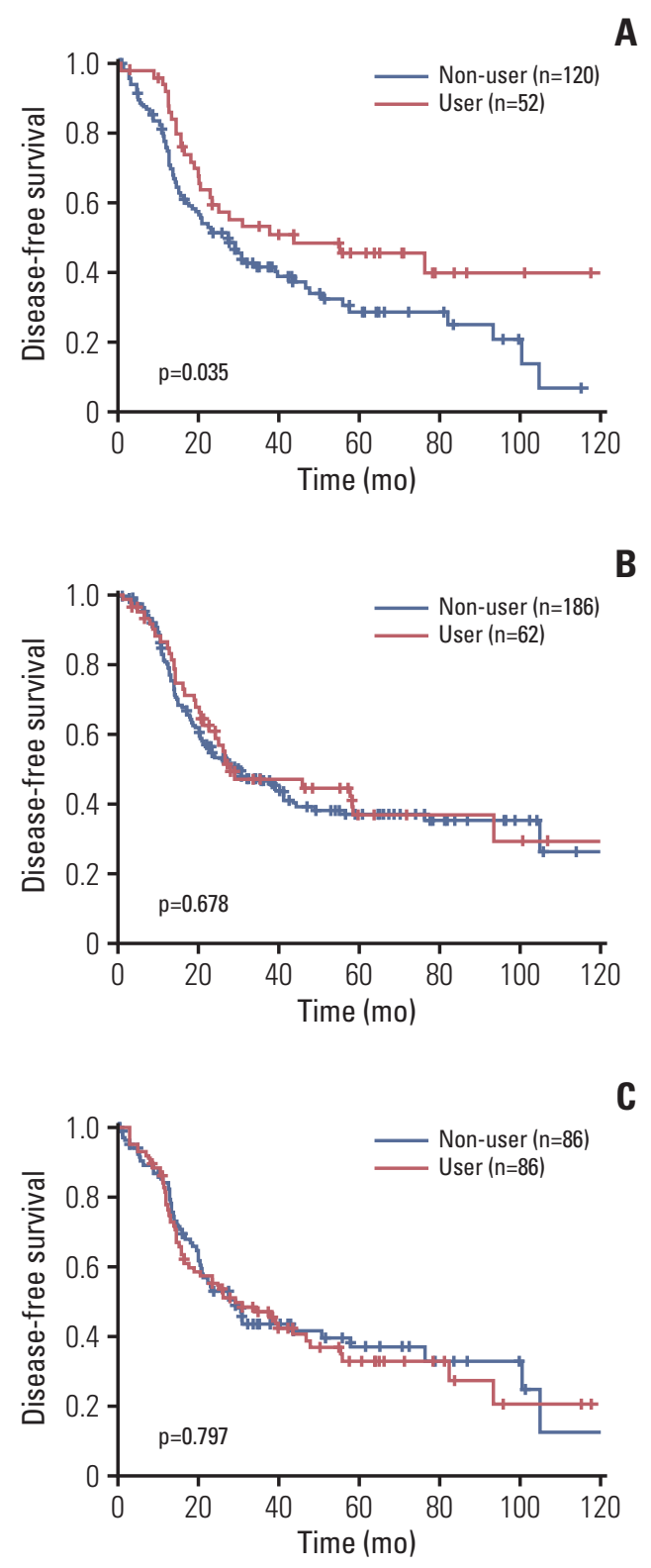

Fig. 2. Kaplan-Meier curve for progression-free survival after propensity score matching in angiotensin receptor blocker group (A), beta blocker group (B), and calciumchannel blocker group (C).

\section{Discussion}

Due to the poor prognosis, investigators have long strived to find novel agents to augment systemic therapies of EOC, as well as identify existing agents that may benefit the efficacy of conventional treatment. While a proposed benefit of ARB in preclinical models has been accumulated, clinical evidence was sparse. This study aimed to disclose difference
Table 4. Multivariate Cox proportional hazards analysis for progression-free survival in matched groups $(\mathrm{n}=878)$

\begin{tabular}{lcc} 
Variable & HR $(95 \%$ CI $)$ & p-value \\
\hline Age $(>50$ yr $)$ & $0.78(0.39-0.93)$ & 0.294 \\
FIGO stage (III /IV) & $2.32(1.38-3.89)$ & 0.001 \\
Residual status (>1 cm) & $1.77(1.19-2.64)$ & 0.005 \\
Comorbidity (yes) & $1.18(0.72-1.94)$ & 0.518 \\
ARBs (use) & $0.60(0.39-0.93)$ & 0.022 \\
BBs (use) & $0.89(0.60-1.31)$ & 0.558 \\
CCBs (use) & $1.17(0.85-1.61)$ & 0.334 \\
\hline
\end{tabular}

$\mathrm{HR}$, hazard ratio; CI, confidence interval; FIGO, International Federation of Gynecology and Obstetrics; ARBs, angiotensin-receptor blockers; BBs, beta blockers; CCBs, calcium channel blockers.

in disease course between patient groups with several types of antihypertensive medications. We conducted a retrospective cohort study to reveal statistically significant interactions between ARB, BB, CCB, and TD use on EOC prognosis.

The survival curves in all enrolled patients did not show statistical relevance regarding $\mathrm{ARB}, \mathrm{BB}$, or TD use. A trend that ARB usage had better PFS was suggested, but was not statistically significant. Only CCB users had higher recurrence rate. Because of significant deviations in age, stage, comorbidities and residual disease, propensity score matching analysis was performed to compensate for disparities in patient groups. In matched groups, patients with ARBs had superior PFS while taking CCB had no relationship with progression. Also, ARB use remained as a significant positive prognostic factor of recurrence in multivariate analysis. Fiftysix patients with ARBs had $40 \%$ lower risk of disease recurrence compared to other patients who did not take ARBs. However, BBs, CCBs, and TDs were not beneficial in terms of cancer recurrence. Conclusively, ARBs were associated with longer disease free duration-even after accounting for other baseline clinical factors. This result is in concordance with the outcomes of previous studies. To this date, in vitro and in vivo studies demonstrated that up-regulation of ACE and angiotensin II type 1 receptor blockers can facilitate local tumor angiogenesis. In turn, several investigators have argued that ARBs may have a potential clinical benefit to target the effects of angiogenic pathways, presumably by inhibiting tumor group and facilitating drug delivery $[3,18]$. In more detail, RAS activation results in enhanced VEGF, which in turn increases angiogenesis and pro-tumorigenic transcription factors. The local RAS system also engages in invasion, migration and metastasis as well as pro-inflammatory process [19]. Recently, tumor-inhibitory ability of telmisartan, an $\mathrm{ARB}$, has been demonstrated by in vivo and in vitro 


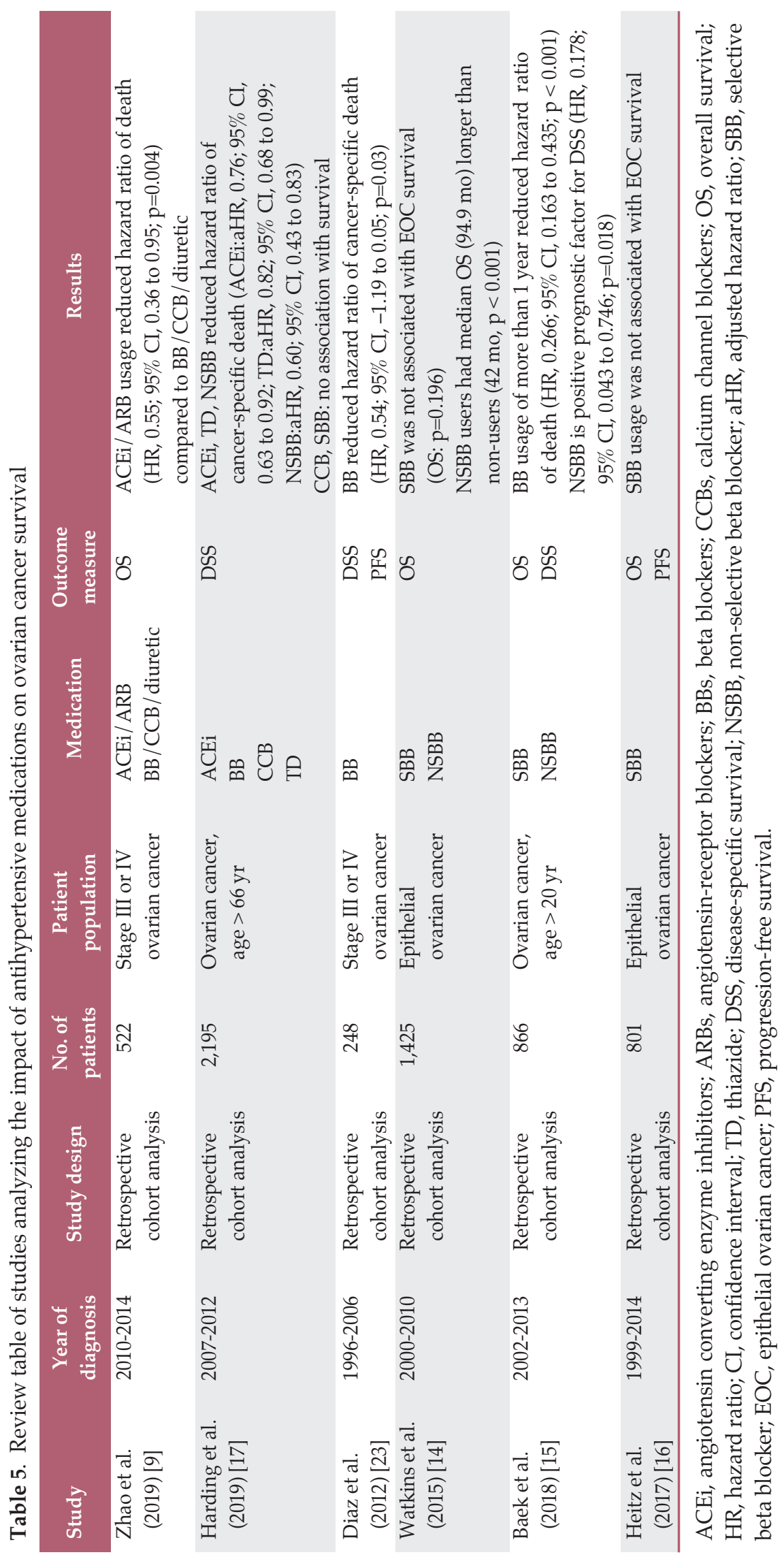


experiments on esophageal squamous cell carcinoma [20]. A remarkable study has reported that losartan, an ARB, enhances drug delivery and efficacy by reducing solid stress and extracellular matrix in ovarian tumors. Their claim was supported by preclinical murine models of ovarian cancer which demonstrated that losartan improved vessel perfusion, which in turn alleviate tumor hypoxia [9]. These findings suggest a physiologic mechanism that explains the result of our study.

Some preclinical studies suggested that stress hormone in the adrenergic system could promote tumor growth by activation of invasive ability and stimulating VEGF secretion [21]. With this concept, some retrospective studies with breast cancer and malignant melanoma experimented if BBs, blockers of adrenergic system, had association with survival and showed that BB users had improved PFS [22]. Previous retrospective cohort studies have also suggested that using beta blockers in EOC patients is associated with longer survival $[14,15,23]$. A pilot study demonstrated the potential role of perioperative propranolol to suppress tumor growth in EOC patients [24]. However, there are several studies with no improved survival in BB users $[16,25]$. In a prospective study with recurrent EOC, BB was not associated with significant impact on PFS and OS [26]. The reasons for these contradictory results are ambiguous, so care should be taken when considering the application of BBs for EOC patients. Several prospective clinical trials on this subject are under investigation. There are two phase 1 studies (NCT02013492 sponsored by William Carson and NCT01308944 sponsored by Washington University School of Medicine) whose main purpose is to determine the effect of beta blockers in EOC survival. A feasibility study (NCT01504126 sponsored by M.D. Anderson Cancer Center) is designed to give propranolol (NSBB) to patients with chemotherapy to treat EOC. Monitoring the conclusions of these trials would be crucial to judge tumor-suppressive effect of BBs.

The role of CCBs in treatment of cancer has also been discussed. Calcium signaling mediates and controls cell cycle progression, such as proliferation, differentiation, growth, and apoptosis and several studies analyzed the cytotoxic effect of CCBs in EOC cell lines $[27,28]$. However, in metaanalysis of role of CCB in several cancers, this drug has been thought to have no association with general cancer prognosis [29]. This study also found that CCBs had no impact on the survival rate of patients with EOC. Like BBs, more clinical studies should be performed to verify the cytotoxic effect of CCBs. Several studies on investigating the effect of antihypertensive medications on EOC are summarized on the review table (Table 5).

This study is one of the first to examine the efficacy of ARB in comparison with $\mathrm{BB}, \mathrm{CCB}$, and $\mathrm{TD}$ in reducing recurrence rate of ovarian cancer. There has been previous research on the impact of several types of antihypertensive medications including ACE inhibitors, BB and CCB on ovarian cancer survival [30], but the clinical implications of ARB treatment for EOC patients has been newly suggested as a possible antineoplastic agent. Moreover, while previous studies were mostly focused on explaining the pathological pathway and expression of angiotensin II receptor, this study reveals the potential role of ARB with significant statistical data in clinical setting.

The major limitation of the study is that dosage, duration, patient adherence of each drug were not included. The patient group with BBs was subdivided into SBBs and NSBBs for more specific verification, but the size of these cohort groups was relatively small. Therefore, limited statistical power can possibly account for the different results in this study compared to previous studies that showed better survival outcomes in NSBB users $[14,15]$. In addition, due to its nonrandomized, retrospective study design, we cannot exclude uncontrolled confounding factors. Even though our sample size allowed adjustment of confounding factors by multivariable analysis, other variables may have affected the conclusion. Therefore, causal relationships still need to be supported by controlled, randomized prospective clinical studies. The inability to obtain the mechanism of each medication is another limitation of this study.

Further investigation is recommended to clarify the significance of ARB use by stratification of dose and duration. We suggest that multi-center clinical trials with a larger population with long-term follow-up should be started.

After controlling for age, stage, comorbidities and cytoreduction status, use of angiotensin-receptor blocker is associated with lower recurrence outcomes in EOC patients. However, CCBs, beta blockers and TDs did not show beneficial impact on progression. Further research is required to verify the differential effect of ARBs in treatment of EOC.

\section{Electronic Supplementary Material}

Supplementary materials are available at Cancer Research and Treatment website (https://www.e-crt.org).

\section{Conflicts of Interest}

Conflict of interest relevant to this article was not reported.

\section{Acknowledgments}

This research was supported by the Basic Science Research Program through the National Research Foundation of Korea (NRF) funded by the Ministry of Education (2017R1D1A1B05035844) and a grant supported by Samsung Medical Center, Republic of Korea (OTC1180321). 


\section{References}

1. Lim MC, Won YJ, Ko MJ, Kim M, Shim SH, Suh DH, et al. Incidence of cervical, endometrial, and ovarian cancer in Korea during 1999-2015. J Gynecol Oncol. 2019;30:e38.

2. Jung KW, Won YJ, Kong HJ, Lee ES. Cancer statistics in Korea: incidence, mortality, survival, and prevalence in 2016. Cancer Res Treat. 2019;51:417-30.

3. Dolley-Hitze T, Jouan F, Martin B, Mottier S, Edeline J, Moranne O, et al. Angiotensin-2 receptors (AT1-R and AT2$\mathrm{R})$, new prognostic factors for renal clear-cell carcinoma? Br J Cancer. 2010;103:1698-705.

4. Roder K, Werdich AA, Li W, Liu M, Kim TY, Organ-Darling LE, et al. RING finger protein RNF207, a novel regulator of cardiac excitation. J Biol Chem. 2014;289:33730-40.

5. Leung PS, Chappell MC. A local pancreatic renin-angiotensin system: endocrine and exocrine roles. Int J Biochem Cell Biol. 2003;35:838-46.

6. Liu WB, Wang XP, Wu K, Zhang RL. Effects of angiotensin II receptor antagonist, Losartan on the apoptosis, proliferation and migration of the human pancreatic stellate cells. World J Gastroenterol. 2005;11:6489-94.

7. Gong Q, Davis M, Chipitsyna G, Yeo CJ, Arafat HA. Blocking angiotensin II type 1 receptor triggers apoptotic cell death in human pancreatic cancer cells. Pancreas. 2010;39:581-94.

8. Diop-Frimpong B, Chauhan VP, Krane S, Boucher Y, Jain RK. Losartan inhibits collagen I synthesis and improves the distribution and efficacy of nanotherapeutics in tumors. Proc Natl Acad Sci U S A. 2011;108:2909-14.

9. Zhao Y, Cao J, Melamed A, Worley M, Gockley A, Jones D, et al. Losartan treatment enhances chemotherapy efficacy and reduces ascites in ovarian cancer models by normalizing the tumor stroma. Proc Natl Acad Sci U S A. 2019;116:2210-9.

10. Hwang SH, Noh SJ, Kwak HJ, Kim KR, Park HS, Moon WS, et al. Expression of angiotensin II receptor type 1 and 2 in ovarian epithelial tumors. Basic Appl Pathol. 2008;1:131-8.

11. Ino K, Shibata K, Kajiyama H, Yamamoto E, Nagasaka T, Nawa A, et al. Angiotensin II type 1 receptor expression in ovarian cancer and its correlation with tumour angiogenesis and patient survival. Br J Cancer. 2006;94:552-60.

12. Suganuma T, Ino K, Shibata K, Kajiyama H, Nagasaka T, Mizutani S, et al. Functional expression of the angiotensin II type 1 receptor in human ovarian carcinoma cells and its blockade therapy resulting in suppression of tumor invasion, angiogenesis, and peritoneal dissemination. Clin Cancer Res. 2005;11:2686-94.

13. Park YA, Choi CH, Do IG, Song SY, Lee JK, Cho YJ, et al. Dual targeting of angiotensin receptors (AGTR1 and AGTR2) in epithelial ovarian carcinoma. Gynecol Oncol. 2014;135:108-17.

14. Watkins JL, Thaker PH, Nick AM, Ramondetta LM, Kumar S, Urbauer DL, et al. Clinical impact of selective and nonselective beta-blockers on survival in patients with ovarian cancer. Cancer. 2015;121:3444-51.

15. Baek MH, Kim DY, Kim SO, Kim YJ, Park YH. Impact of beta blockers on survival outcomes in ovarian cancer: a nationwide population-based cohort study. J Gynecol Oncol. 2018;29:e82.
16. Heitz F, Hengsbach A, Harter P, Traut A, Ataseven B, Schneider $\mathrm{S}$, et al. Intake of selective beta blockers has no impact on survival in patients with epithelial ovarian cancer. Gynecol Oncol. 2017;144:181-6.

17. Harding BN, Delaney JA, Urban RR, Weiss NS. Post-diagnosis use of antihypertensive medications and the risk of death from ovarian cancer. Gynecol Oncol. 2019;154:426-31.

18. Chehl N, Gong Q, Chipitsyna G, Aziz T, Yeo CJ, Arafat HA. Angiotensin II regulates the expression of monocyte chemoattractant protein-1 in pancreatic cancer cells. J Gastrointest Surg. 2009;13:2189-200.

19. Wzgarda A, Kleszcz R, Prokop M, Regulska K, Regulski M, Paluszczak J, et al. Unknown face of known drugs - what else can we expect from angiotensin converting enzyme inhibitors? Eur J Pharmacol. 2017;797:9-19.

20. Matsui T, Chiyo T, Kobara H, Fujihara S, Fujita K, Namima D, et al. Telmisartan inhibits cell proliferation and tumor growth of esophageal squamous cell carcinoma by inducing S-phase arrest in vitro and in vivo. Int J Mol Sci. 2019;20:E3197.

21. Sood AK, Bhatty R, Kamat AA, Landen CN, Han L, Thaker $\mathrm{PH}$, et al. Stress hormone-mediated invasion of ovarian cancer cells. Clin Cancer Res. 2006;12:369-75.

22. Melhem-Bertrandt A, Chavez-Macgregor M, Lei X, Brown EN, Lee RT, Meric-Bernstam F, et al. Beta-blocker use is associated with improved relapse-free survival in patients with triplenegative breast cancer. J Clin Oncol. 2011;29:2645-52.

23. Diaz ES, Karlan BY, Li AJ. Impact of beta blockers on epithelial ovarian cancer survival. Gynecol Oncol. 2012;127:375-8.

24. Jang HI, Lim SH, Lee YY, Kim TJ, Choi CH, Lee JW, et al. Perioperative administration of propranolol to women undergoing ovarian cancer surgery: a pilot study. Obstet Gynecol Sci. 2017;60:170-7.

25. Eskander R, Bessonova L, Chiu C, Ward K, Culver H, Harrison $\mathrm{T}$, et al. Beta blocker use and ovarian cancer survival: a retrospective cohort study. Gynecol Oncol. 2012;127:S21.

26. Heitz F, du Bois A, Harter P, Lubbe D, Kurzeder C, Vergote I, et al. Impact of beta blocker medication in patients with platinum sensitive recurrent ovarian cancer-a combined analysis of 2 prospective multicenter trials by the AGO Study Group, NCIC-CTG and EORTC-GCG. Gynecol Oncol. 2013;129:463-6.

27. Li W, Zhang SL, Wang N, Zhang BB, Li M. Blockade of T-type $\mathrm{Ca}(2+)$ channels inhibits human ovarian cancer cell proliferation. Cancer Invest. 2011;29:339-46.

28. Jang SJ, Choi HW, Choi DL, Cho S, Rim HK, Choi HE, et al. In vitro cytotoxicity on human ovarian cancer cells by T-type calcium channel blockers. Bioorg Med Chem Lett. 2013;23:665662.

29. Sun H, Zhuang RY, Li T, Zheng YT, Cai WM. No association between calcium channel blockers and survival in patients with cancer: a systematic review and meta-analysis. Asian Pac J Cancer Prev. 2016;17:3917-21.

30. Huang T, Sood AK, Tworoger SS. Abstract 4252: Antihypertensive medication use and ovarian cancer survival. Cancer Res. 2018;78:4252. 